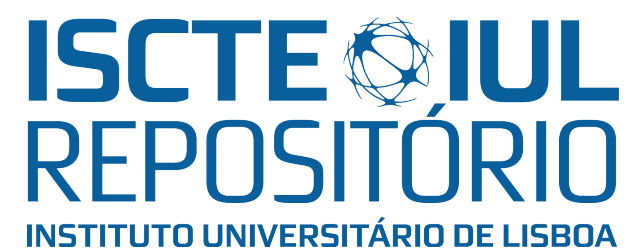

Repositório ISCTE-IUL

Deposited in Repositório ISCTE-IUL:

2019-02-20

Deposited version:

Post-print

Peer-review status of attached file:

Peer-reviewed

Citation for published item:

Bracic, J. \& Diogo, C. (2016). Operators with a given part of the numerical range. Mathematica Slovaca. 66 (1), 275-280

Further information on publisher's website:

10.1515/ms-2015-0134

Publisher's copyright statement:

This is the peer reviewed version of the following article: Bracic, J. \& Diogo, C. (2016). Operators with a given part of the numerical range. Mathematica Slovaca. 66 (1), 275-280, which has been published in final form at https://dx.doi.org/10.1515/ms-2015-0134. This article may be used for non-commercial purposes in accordance with the Publisher's Terms and Conditions for self-archiving.

Use policy

Creative Commons CC BY 4.0

The full-text may be used and/or reproduced, and given to third parties in any format or medium, without prior permission or charge, for personal research or study, educational, or not-for-profit purposes provided that:

- a full bibliographic reference is made to the original source

- a link is made to the metadata record in the Repository

- the full-text is not changed in any way

The full-text must not be sold in any format or medium without the formal permission of the copyright holders. 


\title{
OPERATORS WITH A GIVEN PART OF THE NUMERICAL RANGE
}

\author{
JANKO BRAČIČ* AND CRISTINA DIOGO**
}

\begin{abstract}
Sets of operators which have a given set of numbers in the numerical range are studied. We prove an interesting result which characterizes the set of operators that have 0 in the convex hull of its spectrum. Along the way we give a simpler proof of the well known Hilbebrandt's theorem.
\end{abstract}

\section{INTRODUCTION AND PRELIMINARIES}

Let $\mathscr{H}$ be a complex Hilbert space, $\mathcal{B}(\mathscr{H})$ be the Banach algebra of all bounded linear operators on $\mathscr{H}$, and $I$ be the identity operator. Denote by $\mathcal{S}_{\mathscr{H}}=\{x \in \mathscr{H} ;\|x\|=1\}$ the unit sphere of $\mathscr{H}$. The numerical range of $A \in \mathcal{B}(\mathscr{H})$ is

$$
W(A)=\left\{\langle A x, x\rangle ; x \in \mathcal{S}_{\mathscr{H}}\right\} .
$$

It is obvious that $W(A)$ is a non-empty subset of $\mathbb{C}$ which is contained in the closed disk $\overline{\mathbb{D}(0,\|A\|)}=\{z \in \mathbb{C} ;|z| \leq\|A\|\}$. If $\operatorname{dim}(\mathscr{H})<\infty$, then $W(A)$ is a closed set. However, if $\mathscr{H}$ is not finite-dimensional, then the numerical range is not closed, in general. For instance, the numerical range of the backward shift on $\ell^{2}$ is the open unit disk $\mathbb{D}(0,1)$.

If $A^{*} \in \mathcal{B}(\mathscr{H})$ is the adjoint operator of $A \in \mathcal{B}(\mathscr{H})$, then $W\left(A^{*}\right)=\{\bar{\lambda} ; \lambda \in$ $W(A)\}$. Thus, an operator $A$ is selfadjoint if and only if its numerical range consists of real numbers.

One among the basic properties of the numerical range is its convexity.

Theorem 1.1. For every $A \in \mathcal{B}(\mathscr{H})$, the numerical range $W(A)$ is a convex set.

The proof of this statement, which is usually called The Toeplitz-Hausdorff Theorem, relies on the following two results. The first is The Elliptical Range

2010 Mathematics Subject Classification. Primary 47A12.

Key words and phrases. Numerical Range, Hildebrandt's Theorem.

The work was partially supported by FCT through the Program POCI 2010/FEDER and the project PTDC/MAT/121837/2010, Portugal. 
Theorem which gives the complete description of the numerical range of a 2-by-2 matrix and can be stated as follows.

Theorem 1.2. The numerical range of $A=\left[\begin{array}{cc}\lambda & \omega \\ 0 & \mu\end{array}\right]$ is the elliptical disk with foci at $\lambda$ and $\mu$, the eigenvalues of $A$, and with the semi-axes

$$
a=\frac{1}{2} \sqrt{|\omega|^{2}+|\lambda-\mu|^{2}} \quad \text { and } \quad b=\frac{1}{2}|\omega| .
$$

The second result is that the numerical range of any compression of $A \in \mathcal{B}(\mathscr{H})$ is included in $W(A)$, that is, if $\mathscr{M}$ is a closed subspace of $\mathscr{H}$ and $P$ is the orthogonal projection on $\mathscr{M}$, then $W(P A P) \subseteq W(A)$. Note that $P A P$ is considered as an operator on $\mathscr{M}$.

More information about numerical ranges can be found in [1] and [2], for instance.

The aim of this paper is to study sets of operators with a prescribed part of the numerical range. More precisely, we are interested in sets $\mathscr{W}_{E}=\{A \in$ $\mathcal{B}(\mathscr{H}) ; E \subseteq \overline{W(A)}\}$, where $E \subseteq \mathbb{C}$ is a given set. In Section 2, we present some basic properties of these sets. It is shown, through an example, that we cannot expect that $\mathscr{W}_{E}$ carries a "usual" algebraic structure; for instance, it is not closed for addition or multiplication. However, we give an example when $\mathscr{W}_{E}$ has a nice and interesting algebraic property: we are able to characterize the set of all operators $A$ such that $0 \in \overline{W(P A)}$ for every positive semidefinite operator $P$. In the proof of our main result, we use Hildebrandt's Theorem. This result is usually proved by The Rota Theorem, which says that every strict contraction on a Hilbert space is similar to a part of a backward shift (see [7], for instance). In the rest of this section, we present a different and simpler proof of Hildebrant's Theorem which relies on a lemma proved by Murphy and West.

Let $\sigma(A)$ be the spectrum of $A \in \mathcal{B}(\mathscr{H})$ and $r(A)$ be the spectral radius. For an arbitrary set $E \subseteq \mathbb{C}$, let $\operatorname{conv}(E)$ be the convex hull of $E$. It is easily seen that every eigenvalue of $A$ is in $W(A)$, which in the finite-dimensional case already gives $\operatorname{conv}(\sigma(A)) \subseteq W(A)$. This inclusion, with $W(A)$ replaced by its closure, actually holds for every operator. To prove it one has to invoke the notion of approximate eigenvalues and use the fact that each point in the boundary $\partial \sigma(A)$ is an approximate eigenvalue for $A$. Since the spectrum is preserved by similarities one has

$$
\operatorname{conv}(\sigma(A)) \subseteq \bigcap_{\substack{S \in \mathcal{B}(\mathscr{H}) \\ \text { invertible }}} \overline{W\left(S A S^{-1}\right)},
$$

for every $A \in \mathcal{B}(\mathscr{H})$. Hildebrandt [3] observed that (1.1) is actually an equality.

The following lemma is from [6]; for the sake of completeness we include its proof.

Lemma 1.1. For $A \in \mathcal{B}(\mathscr{H})$ and $\varepsilon>0$, there exists an invertible operator $S$ such that $\left\|S A S^{-1}\right\|<r(A)+\varepsilon$. 
Proof. Let $B=\frac{1}{r(A)+\varepsilon} A$. Then $r(B)<1$, that is, $\lim _{n \rightarrow \infty}\left\|B^{n}\right\|^{1 / n}<1$, using the Gelfand-Beurling formula (see [5, Theorem I.22]). Hence, the series $\sum_{n=0}^{\infty}\left\|\left(B^{n}\right)^{*} B^{n}\right\|=\sum_{n=0}^{\infty}\left\|B^{n}\right\|^{2}$ converges - use the Cauchy root test. It follows that $C=\sum_{n=0}^{\infty}\left(B^{n}\right)^{*} B^{n} \in \mathcal{B}(\mathscr{H})$ and $C \geq I$. Let $S=\sqrt{C}$. Then $S \geq I$, which means that it is invertible. Since $0 \leq I-S^{-2} \leq I$, we have

$$
\begin{aligned}
\left\|S B S^{-1}\right\|^{2} & =\left\|S^{-1} B^{*} S^{2} B S^{-1}\right\|=\left\|S^{-1} \sum_{n=1}^{\infty}\left(B^{n}\right)^{*} B^{n} S^{-1}\right\| \\
& =\left\|S^{-1}\left(S^{2}-I\right) S^{-1}\right\|=\left\|I-S^{-2}\right\|=r\left(I-S^{-2}\right)<1 .
\end{aligned}
$$

It is obvious now that $\left\|S A S^{-1}\right\|<r(A)+\varepsilon$.

Theorem 1.3 (Hildebrandt's Theorem). For every $A \in \mathcal{B}(\mathscr{H})$,

$$
\operatorname{conv}(\sigma(A))=\bigcap_{\substack{S \in \mathcal{B}(\mathscr{H}) \\ \text { invertible }}} \overline{W\left(S A S^{-1}\right)} .
$$

Proof. One inclusion has already been established in (1.1). To prove the other inclusion, let $\lambda \in \mathbb{C} \backslash \operatorname{conv}(\sigma(A))$. Since $\operatorname{conv}(\sigma(A))$ is a compact convex set there exists a disk $\mathbb{D}(\mu, \rho)$ such that $\lambda \notin \overline{\mathbb{D}(\mu, \rho)}$ and $\operatorname{conv}(\sigma(A)) \subseteq \mathbb{D}(\mu, \rho)$. Hence $\lambda-\mu \notin \overline{\mathbb{D}(0, \rho)}$ and $\operatorname{conv}(\sigma(A-\mu I)) \subseteq \mathbb{D}(0, \rho)$, which means that $r(A-\mu I)<\rho$. Let $\varepsilon>0$ be such that $r(A-\mu I)+\varepsilon<\rho$. By Lemma 1.1, there exists an invertible operator $S$ such that $\left\|S(A-\mu I) S^{-1}\right\|<r(A-\mu I)+\varepsilon<\rho$. It follows that $\lambda-\mu \notin \overline{W\left(S(A-\mu I) S^{-1}\right)}$ and consequently $\lambda \notin \overline{W\left(S A S^{-1}\right)}$.

\section{Sets of operators With a given Set in the nUmerical Range}

For a set $E \subseteq \mathbb{C}$, let $\mathscr{W}_{E}$ be the collection of all operators which contain $E$ in the closure of the numerical range, that is,

$$
\mathscr{W}_{E}=\{A \in \mathcal{B}(\mathscr{H}) ; E \subseteq \overline{W(A)}\} .
$$

It is obvious that $\mathscr{W}_{\emptyset}=\mathcal{B}(\mathscr{H})$ and that $\mathscr{W}_{E}=\emptyset$ if and only if $E$ is an unbounded set. Note that $\mathscr{W}_{E_{1}} \supseteq \mathscr{W}_{E_{2}}$ whenever $E_{1} \subseteq E_{2}$; in particular, $\mathscr{W}_{E} \supseteq \mathscr{W}_{\overline{\operatorname{conv}(E)}}$. However, since $\overline{\operatorname{conv}(E)} \subseteq \overline{W(A)}$ if $E \subseteq \overline{W(A)}$ one has $\mathscr{W}_{E}=\mathscr{W}_{\operatorname{conv}(E)}$. Therefore we may assume without loss of generality that $E$ is a bounded, closed and convex set. Let $\mathscr{C}(\mathbb{C})$ be the family of all bounded, closed and convex subsets of $\mathbb{C}$. It is easily seen that $\mathscr{C}(\mathbb{C})$ is a lattice for the operations

$$
E_{1} \wedge E_{2}=E_{1} \cap E_{2} \quad \text { and } \quad E_{1} \vee E_{2}=\operatorname{conv}\left(E_{1} \cup E_{2}\right),
$$


where $E_{1}, E_{2} \in \mathscr{C}(\mathbb{C})$ are arbitrary. Let $\mathcal{W}=\left\{\mathscr{W}_{E} ; \quad E \in \mathscr{C}(\mathbb{C})\right\}$. Then $\mathcal{W}$ has a natural lattice structure, which is given by

$$
\mathscr{W}_{E_{1}} \vee \mathscr{W}_{E_{2}}=\mathscr{W}_{E_{1} \wedge E_{2}} \quad \text { and } \quad \mathscr{W}_{E_{1}} \wedge \mathscr{W}_{E_{2}}=\mathscr{W}_{E_{1} \vee E_{2}}
$$

Proposition 2.1. For every $E \in \mathscr{C}(\mathbb{C})$, the set $\mathscr{W}_{E}$ is nonempty and uniformly closed.

Proof. It is obvious that $\mathscr{W}_{E}$ is nonempty. Let $\left\{A_{n}\right\}_{n=1}^{\infty} \subseteq \mathscr{W}_{E}$ be a sequence which converges to $A \in \mathcal{B}(\mathscr{H})$ and let $\lambda \in E$. If $\varepsilon>0$, then there exists an index $n_{\varepsilon}$ such that $\left\|A-A_{n}\right\|<\varepsilon$ for all $n \geq n_{\varepsilon}$. Let $n_{0} \geq n_{\varepsilon}$ and let $x \in \mathcal{S}_{\mathscr{H}}$ be such that $\left|\left\langle A_{n_{0}} x, x\right\rangle-\lambda\right|<\varepsilon$. It follows that $|\langle A x, x\rangle-\lambda| \leq$ $\left|\langle A x, x\rangle-\left\langle A_{n_{0}} x, x\right\rangle\right|+\left|\left\langle A_{n_{0}} x, x\right\rangle-\lambda\right|<2 \varepsilon$. Since $\varepsilon$ is arbitrary we may conclude that $\lambda \in \overline{W(A)}$ and consequently $E \subseteq \overline{W(A)}$.

Let $k$ be a positive integer. A set $\mathcal{S} \subseteq \mathcal{B}(\mathscr{H})$ is said to be $k$-transitive if for every linearly independent vectors $x_{1}, \ldots, x_{k} \in \mathscr{H}$ and for every set of $k$ vectors $\left\{y_{1}, \ldots, y_{k}\right\} \subseteq \mathscr{H}$ there exists an operator $S \in \mathcal{S}$ such that $S x_{i}=y_{i}$ $(i=1, \ldots, k)$.

Proposition 2.2. Let $E \in \mathscr{C}(\mathbb{C})$. If $\operatorname{dim}(\mathscr{H}) \geq k+2$, then $\mathscr{W}_{E}$ is $k$-transitive.

Proof. Let $x_{1}, \ldots, x_{k} \in \mathscr{H}$ be linearly independent and let $\left\{y_{1}, \ldots, y_{k}\right\} \subseteq \mathscr{H}$ be an arbitrary set of $k$ vectors. Since $\operatorname{dim}(\mathscr{H}) \geq k+2$ there exist orthogonal vectors $e_{1}, e_{2} \in \mathcal{S}_{\mathscr{H}}$ such that $x_{i} \perp e_{j}(i=1, \ldots, k$ and $j=1,2)$. Denote by $\mathscr{H}_{1}$ the linear span of $\left\{e_{1}, e_{2}\right\}$ and let $P$ be the orthogonal projection onto $\mathscr{H}_{1}$. Let $\lambda, \mu, \omega \in \mathbb{C}$ be such that the elliptical disk $\mathscr{E}$ with foci at $\lambda$ and $\mu$ and with semiaxes $a=\frac{1}{2} \sqrt{|\omega|^{2}+|\lambda-\mu|^{2}}$ and $b=\frac{1}{2}|\omega|$ contains $E$. Since $e_{1}, e_{2}, x_{1}, \ldots, x_{k}$ are linearly independent there exists $A \in \mathcal{B}(\mathscr{H})$ such that $A e_{1}=\lambda e_{1}, A e_{2}=$ $\omega e_{1}+\mu e_{2}$ and $A x_{i}=y_{i}(i=1, \ldots, k)$. By Theorem 1.2, the numerical range of the compression $P A P$ is the disk $\mathscr{E}$. Thus, $E \subseteq W(P A P) \subseteq W(A)$ and therefore $A \in \mathscr{W}_{E}$.

Let $\mathscr{F}: \mathbb{C} \rightarrow \mathbb{C}$ be an affine transformation which is given by

$$
\mathscr{F}: u+i v \mapsto a u+b v+e+i(c u+d v+f) \quad(u, v \in \mathbb{R}),
$$

where $a, b, c, d, e, f$ are real numbers such that $a d-b c \neq 0$. It is easily seen that $\mathscr{F}$ is bijective and that its inverse $\mathscr{F}^{-1}$ is an affine transformation as well. Mappings $\mathscr{F}$ and $\mathscr{F}^{-1}$ are continuous, thus $\mathscr{F}$ maps open sets into open sets and closed sets into closed sets. It follows that $\mathscr{F}(\bar{E})=\overline{\mathscr{F}(E)}$, for every nonempty set $E \subseteq \mathbb{C}$.

For $A \in \mathcal{B}(\mathscr{H})$, let $A=H+i K$ be its Cartesian decomposition. If $\mathscr{F}$ is given by $(2.2)$, then $\mathscr{F}(A)$ is defined by

$$
\mathscr{F}(A)=a H+b K+e I+i(c H+d K+f I) .
$$

It is easily seen that the following proposition holds. 
Proposition 2.3. If $\mathscr{F}$ is an affine transformation, then $W(\mathscr{F}(A))=\mathscr{F}(W(A))$, for any $A \in \mathcal{B}(\mathscr{H})$.

Taking this into account, we have the following result.

Proposition 2.4. Let $E \in \mathscr{C}(\mathbb{C})$. If $\mathscr{F}$ is an affine transformation, then $\mathscr{W}_{\mathscr{F}(E)}=\mathscr{F}\left(\mathscr{W}_{E}\right)$.

Proof. If $A \in \mathscr{W}_{E}$, then $\mathscr{F}(E) \subseteq \mathscr{F}(\overline{W(A)})=\overline{W(\mathscr{F}(A))}$, by Proposition 2.3. Therefore, $\mathscr{F}(A) \in \mathscr{W}_{\mathscr{F}(E)}$. On the other hand, if $A \in \mathscr{W}_{\mathscr{F}(E)}$, using Proposition 2.3 again, we have $E=\mathscr{F}^{-1}(\mathscr{F}(E)) \subseteq \mathscr{F}^{-1}(\overline{W(A)})=\overline{\mathscr{F}-1(W(A))}=$ $\overline{W(\mathscr{F}-1(A))}$. Thus, $\mathscr{F}^{-1}(A) \in \mathscr{W}_{E}$ and therefore $A \in \mathscr{F}\left(\mathscr{W}_{E}\right)$.

Lemma 2.1. Let $\mathscr{F}$ be an affine transformation. Then $\mathscr{F}(E) \in \mathscr{C}(\mathbb{C})$, for every $E \in \mathscr{C}(\mathbb{C})$. Moreover, $\mathscr{F}$ is a lattice isomorphism of $\mathscr{C}(\mathbb{C})$, which means that

$$
\mathscr{F}\left(E_{1} \wedge E_{2}\right)=\mathscr{F}\left(E_{1}\right) \wedge \mathscr{F}\left(E_{2}\right) \quad \text { and } \quad \mathscr{F}\left(E_{1} \vee E_{2}\right)=\mathscr{F}\left(E_{1}\right) \vee \mathscr{F}\left(E_{2}\right)
$$

for all $E_{1}, E_{2} \in \mathscr{C}(\mathbb{C})$.

Proof. Let $\mathscr{F}$ be given by $(2.2)$. If $E \in \mathscr{C}(\mathbb{C})$ and $u_{1}+i v_{1}, u_{2}+i v_{2} \in E$, then $\mathscr{F}\left(t\left(u_{1}+i v_{1}\right)+(1-t)\left(u_{2}+i v_{2}\right)\right)=t \mathscr{F}\left(u_{1}+i v_{1}\right)+(1-t) \mathscr{F}\left(u_{2}+i v_{2}\right)$, for every $t \in[0,1]$. Hence $\mathscr{F}(E) \in \mathscr{C}(\mathbb{C})$.

Let $E_{1}, E_{2} \in \mathscr{C}(\mathbb{C})$ be arbitrary nonempty sets. It is easily seen that $\mathscr{F}\left(E_{1} \wedge\right.$ $\left.E_{2}\right)=\mathscr{F}\left(E_{1}\right) \wedge \mathscr{F}\left(E_{2}\right)$. If $u+i v \in E_{1} \vee E_{2}$, then there exist $u_{1}+i v_{1} \in E_{1}$, $u_{2}+i v_{2} \in E_{2}$, and $t \in[0,1]$ such that $u+i v=t\left(u_{1}+i v_{1}\right)+(1-t)\left(u_{2}+i v_{2}\right)$. Hence $\mathscr{F}(u+i v)=t \mathscr{F}\left(u_{1}+i v_{1}\right)+(1-t) \mathscr{F}\left(u_{2}+i v_{2}\right) \in \mathscr{F}\left(E_{1}\right) \vee \mathscr{F}\left(E_{2}\right)$. The opposite inclusion is proved similarly by using the inverse of $\mathscr{F}$.

Proposition 2.5. Every affine map $\mathscr{F}$ is a lattice isomorphism of $\mathcal{W}$, that is, for every $E_{1}, E_{2} \in \mathscr{C}(\mathbb{C})$,

$\mathscr{F}\left(\mathscr{W}_{E_{1}} \wedge \mathscr{W}_{E_{2}}\right)=\mathscr{F}\left(\mathscr{W}_{E_{1}}\right) \wedge \mathscr{F}\left(\mathscr{W}_{E_{2}}\right)$ and $\mathscr{F}\left(\mathscr{W}_{E_{1}} \vee \mathscr{W}_{E_{2}}\right)=\mathscr{F}\left(\mathscr{W}_{E_{1}}\right) \vee \mathscr{F}\left(\mathscr{W}_{E_{2}}\right)$

Proof. By (2.1), Proposition 2.4 and Lemma 2.1, one has

$$
\begin{aligned}
\mathscr{F}\left(\mathscr{W}_{E_{1}} \wedge \mathscr{W}_{E_{2}}\right) & =\mathscr{F}\left(\mathscr{W}_{E_{1} \vee E_{2}}\right)=\mathscr{W}_{\mathscr{F}\left(E_{1} \vee E_{2}\right)}=\mathscr{W}_{\mathscr{F}\left(E_{1}\right) \vee \mathscr{F}\left(E_{2}\right)} \\
& =\mathscr{W}_{\mathscr{F}\left(E_{1}\right)} \wedge \mathscr{W}_{\mathscr{F}\left(E_{2}\right)}=\mathscr{F}\left(\mathscr{W}_{E_{1}}\right) \wedge \mathscr{F}\left(\mathscr{W}_{E_{2}}\right) .
\end{aligned}
$$

The second equality is proved similarly.

Now we are looking for an algebraic structure of $\mathscr{W}_{E}$. However it is not hard to see that $\mathscr{W}_{E}$ cannot be closed for addition or multiplication. For instance, assume that $E \subseteq \overline{\mathbb{D}(0,1)}$. This is not a big restriction as, by Proposition 2.4, $E$ can be replaced by $\lambda E+\mu$, where $\lambda \neq 0, \mu$ are arbitrary complex numbers. Let $e, f \in \mathcal{S}_{\mathscr{H}}$ be orthogonal vectors and let $M, N \in \mathcal{B}(\mathscr{H})$ be defined by $M e=0=N f, M f=2 e, N e=2 f$, and let they be zero on the orthogonal complement of $\{e, f\}$. Note that $M$ and $N$ are unitarily equivalent. Since 
$W(M)=W(N)=\overline{\mathbb{D}(0,1)}$ we have $M, N \in \mathscr{W}_{E}$. However, $M+N$ is selfadjoint with $W(M+N)=[-2,2]$ and $M^{2}=0$. Hence, $M+N$ and $M^{2}$ are in $\mathscr{W}_{E}$ only in a very particular cases of $E$.

Now we will look for an algebraic structure of $\mathscr{W}_{E}$ which is given in the following sense. Let $\mathcal{P} \subseteq \mathcal{B}(\mathscr{H})$ be a given set of operators. For $E \in \mathscr{C}(\mathbb{C})$, determine the largest sets $\mathcal{Q}(\mathcal{P}, E), \mathcal{R}(\mathcal{P}, E) \subseteq \mathcal{B}(\mathscr{H})$ such that

$$
\mathcal{P} \mathcal{Q}(\mathcal{P}, E) \subseteq \mathscr{W}_{E} \quad \text { and } \quad \mathcal{R}(\mathcal{P}, E) \mathcal{P} \subseteq \mathscr{W}_{E},
$$

where $\mathcal{P} \mathcal{Q}(\mathcal{P}, E)$ is the set of all products $P Q$ with $P \in \mathcal{P}$ and $Q \in \mathcal{Q}(\mathcal{P}, E)$ and $\mathcal{R}(\mathcal{P}, E) \mathcal{P}$ has a similar meaning. It follows from the part (i) of the following proposition that it is enough to study only one variant of the problem. We use the following notation: for $E \in \mathscr{C}(\mathbb{C})$ let $E^{*}=\{\bar{\lambda} ; \lambda \in E\}$ and for $\mathcal{A} \subseteq \mathcal{B}(\mathscr{H})$ let $\mathcal{A}^{*}=\left\{A^{*} ; A \in \mathcal{A}\right\}$.

Proposition 2.6. Let $E, E_{1}, E_{2} \in \mathscr{C}(\mathbb{C})$ and $\mathcal{P}, \mathcal{P}_{1}, \mathcal{P}_{2} \subseteq \mathcal{B}(\mathscr{H})$ be arbitrary. Then
(i) $\mathcal{Q}(\mathcal{P}, E)^{*}=\mathcal{R}\left(\mathcal{P}^{*}, E^{*}\right)$;
(ii) if $I \in \mathcal{P}$, then $\mathcal{Q}(\mathcal{P}, E) \subseteq \mathscr{W}_{E}$;
(iii) if $0 \in \mathcal{P}$, then $\mathcal{Q}(\mathcal{P}, E)=\emptyset$ whenever $E \nsubseteq\{0\}$;
(iv) $\mathcal{Q}(\mathcal{P}, \emptyset)=\mathcal{B}(\mathscr{H})$;
(v) if $E_{1} \subseteq E_{2}$, then $\mathcal{Q}\left(\mathcal{P}, E_{1}\right) \supseteq \mathcal{Q}\left(\mathcal{P}, E_{2}\right)$;
(vi) if $\mathcal{P}_{1} \subseteq \mathcal{P}_{2}$, then $\mathcal{Q}\left(\mathcal{P}_{1}, E\right) \supseteq \mathcal{Q}\left(\mathcal{P}_{2}, E\right)$.

Proof. We prove only (i) as the rest is easily seen. First observe that $\mathscr{W}_{E}^{*}=\mathscr{W}_{E^{*}}$. Thus, one has

$$
\begin{aligned}
A \in \mathcal{Q}(\mathcal{P}, E) & \Longleftrightarrow P A \in \mathscr{W}_{E}(\forall P \in \mathcal{P}) \Longleftrightarrow \\
& \Longleftrightarrow A^{*} P^{*} \in \mathscr{W}_{E^{*}}\left(\forall P^{*} \in \mathcal{P}^{*}\right) \Longleftrightarrow A^{*} \in \mathcal{R}\left(\mathcal{P}^{*}, E^{*}\right) .
\end{aligned}
$$

Let $\mathcal{B}_{+}=\{P \in \mathcal{B}(\mathscr{H}) ; P \geq 0\}$ be the set of all positive semidefinite operators on $\mathscr{H}$. By statements (iii) and (iv) of Proposition 2.6, the only interesting case to consider $\mathcal{Q}\left(\mathcal{B}_{+}, E\right)$ is for $E=\{0\}$.

Theorem 2.7. $\mathcal{Q}\left(\mathcal{B}_{+},\{0\}\right)=\{A \in \mathcal{B}(\mathscr{H}) ; 0 \in \operatorname{conv}(\sigma(A))\}$.

In the proof we need the following simple lemma. If $F \subseteq \mathbb{C}$ is a nonempty set and $w \in \mathbb{C}$, then let $\operatorname{dist}(w, F)=\inf \{|w-z| ; z \in F\}$ and, for $\varepsilon>0$, let $F_{\varepsilon}=\{w \in \mathbb{C} ; \operatorname{dist}(w, F) \leq \varepsilon\}$ denote the $\varepsilon$-hull of $F$.

Lemma 2.2. If $\emptyset \neq F \subseteq \mathbb{C}$, then $\bigcap_{\varepsilon>0} F_{\varepsilon}=\bar{F}$.

Proof. It is obvious that $\bar{F} \subseteq F_{\varepsilon}$ for every $\varepsilon>0$. If $w \in \mathbb{C} \backslash \bar{F}$, then there exists $r>0$ such that $\mathbb{D}(w, r) \subseteq \mathbb{C} \backslash \bar{F}$. It is clear that $w \notin F_{r / 2}$. 
Proof of Theorem 2.7. Suppose that $0 \in \operatorname{conv}(\sigma(A))$. If $A$ or $P$ is not invertible, then $0 \in \sigma(P A) \subseteq \overline{W(P A)}$. Assume therefore that $A$ and $P$ are invertible. It follows that there exists $p>0$ such that $\overline{W(P)} \subseteq[p, \infty)$. Since $0 \in \operatorname{conv}(\sigma(A))$ there exist $\lambda, \mu \in \partial \sigma(A)$ such that $0=t \lambda+(1-t) \mu$ for some $t \in[0,1]$. Numbers $\lambda$ and $\mu$ are approximate eigenvalues of $A$, which means that there exist sequences $\left\{e_{n}\right\}_{n=1}^{\infty}, \quad\left\{f_{n}\right\}_{n=1}^{\infty} \subseteq \mathcal{S}_{\mathscr{H}}$ such that $\lim _{n \rightarrow \infty}\left\|(A-\lambda I) e_{n}\right\|=0$ and $\lim _{n \rightarrow \infty} \|(A-$ $\mu I) f_{n} \|=0$. Let $m$ be a positive integer. Then there exists an index $n_{m}$ such that $\left\|(A-\lambda I) e_{n}\right\|<\frac{1}{m}$ and $\left\|(A-\mu I) f_{n}\right\|<\frac{1}{m}$ for all $n \geq n_{m}$. Fix $n \geq n_{m}$ and denote $\omega_{m}=\left\langle P e_{n}, e_{n}\right\rangle, \vartheta_{m}=\left\langle P f_{n}, f_{n}\right\rangle$. Note that $\omega_{m} \geq p$ and $\vartheta_{m} \geq p$. One has

$$
\left|\left\langle P A e_{n}, e_{n}\right\rangle-\lambda \omega_{m}\right|=\left|\left\langle P(A-\lambda I) e_{n}, e_{n}\right\rangle\right| \leq\|P\|\left\|(A-\lambda I) e_{n}\right\|<\frac{\|P\|}{m}
$$

and, similarly, $\left|\left\langle P A f_{n}, f_{n}\right\rangle-\mu \vartheta_{m}\right|<\|P\| / m$. Thus, $\lambda \omega_{m}$ and $\mu \vartheta_{m}$ are in the $\frac{\|P\|}{m}$-hull of $\overline{W(P A)}$. Since $\left\{\lambda \omega_{m}\right\}_{m=1}^{\infty}$ is a bounded sequence there exists a convergent subsequence, say $\left\{\lambda \omega_{m_{k}}\right\}_{k=1}^{\infty}$, which converges to $\lambda \omega$. It is obvious that this limit is in $\overline{W(P A)}$. Observe that $\omega \geq p$. The same reasoning gives $\vartheta \geq p$ such that $\mu \vartheta \in \overline{W(P A)}$. Denote $s_{1}=t \vartheta /(t \vartheta+(1-t) \omega) \geq 0$ and $s_{2}=(1-t) \omega /(t \vartheta+(1-t) \omega) \geq 0$. It is easily seen that $s_{1}+s_{2}=1$ and $s_{1}(\lambda \omega)+s_{2}(\mu \vartheta)=0$, which means that $0 \in \overline{W(P A)}$.

Assume now that $A \in \mathcal{Q}\left(\mathcal{B}_{+},\{0\}\right)$. Let $S \in \mathcal{B}(\mathscr{H})$ be an arbitrary invertible operator. Denote $P=S^{*} S \in \mathcal{B}_{+}$. Let $\varepsilon>0$ be arbitrary. Since $0 \in \overline{W(P A)}$ there exists $x \in \mathcal{S}_{\mathscr{H}}$ (which may depend on $\varepsilon$ ) such that $|\langle P A x, x\rangle|<\varepsilon$. Let $y=\frac{1}{\|S x\|} S x \in \mathcal{S}_{\mathscr{H}}$. One has $\left|\left\langle S A S^{-1} y, y\right\rangle\right|=\|S x\|^{-2}\left|\left\langle S A S^{-1} S x, S x\right\rangle\right|=$ $\|S x\|^{-2}|\langle P A x, x\rangle|<\|S x\|^{-2} \varepsilon \leq\left\|S^{-1}\right\|^{2} \varepsilon$. Since $\varepsilon$ is arbitrary we conclude that $0 \in \overline{W\left(S A S^{-1}\right)}$. As $S$ is an arbitrary invertible operator we have, by the Hildebrandt's Theorem, $0 \in \operatorname{conv}(\sigma(A))$.

Acknowledgement. We would like to thank the referee for valuable comments and suggestions.

\section{REFERENCES}

[1] Gustafson, K. E. - RAO, D. K. M.: Numerical Range, Springer-Verlag, New York, 1997.

[2] Halmos, P. R.: A Hilbert Space Problem Book, Springer-Verlag, New-York, 1982.

[3] HILDEBRANDT, S.: Über den Numerischen Wertebereich eines Operators, Math. Ann. 163 (1966) 230-247.

[4] LAURSEN, K. B. - NEUMANN, M. N.: An Introduction to Local Spectral Theory, London Math. Soc. Monographs 20, Clarendon Press, Oxford, 2000.

[5] MÜLLER, V.: Spectral Theory of Linear Operators and Spectral Systems in Banach Algebras, Second Edition, Operator Theory: Advances and Applications, Vol. 139, Birkhäuser, 2007.

[6] MURPhY, G. J. - WEST, T. T.: Spectral radius formulae, Proc. Edinburgh Math. Soc. 22 (1979) 271-275. 
[7] WILliAMS, J.: Similarity and the numerical range, J. Math. Anal. App. 26 (1969) 307-314.

* University of Ljubluana

IMFM, JADRANSKA UL. 19

SI-1000 LJUBLJANA

SLOVENIA

E-mail address: janko.bracic@fmf.uni-lj.si

** Instituto Universitário de Lisboa (ISCTE-IUL)

Avenida das Forças Armadas

P-1649-026 LISBOA

PORTUGAL

AND

Center for Mathematical Analysis, Geometry, and Dynamical Systems

Departamento de Matemática

InSTITUTO Superior TÉCNICO

P-1049-001 LISBOA

PORTUGAL

E-mail address: cristina.diogo@iscte.pt 